\title{
EFECTOS DE LA FRAGMENTACIÓN DEL MATORRAL DESÉRTICO SOBRE POBLACIONES DEL AGUILILLA COLA-ROJA Y EL CERNÍCALO AMERICANO EN BAJA CALIFORNIA SUR, MÉXICO
}

\author{
ROMEO TINAJERO \& RICARDO RODRÍGUEZ-ESTRELLA \\ Centro de Investigaciones Biológicas del Noroeste (CIBNOR). Mar Bermejo 195, Col. Playa Palo de \\ Santa Rita, La Paz, Baja California Sur, 23090, México. <jromeoti@gmail.com>; <estrella@cibnor.mx>
}

Tinajero, R. \& R. Rodríguez-Estrella. 2012. Efectos de la fragmentación del matorral desértico sobre poblaciones del aguililla cola-roja y el cernícalo americano en Baja California Sur, México. Acta Zoológica Mexicana (n. s.), 28(2): 427-446.

RESUMEN. Para evaluar el efecto de la fragmentación del desierto sobre especies de poblaciones residentes y migratorias, durante 2008 y 2009 se realizaron muestreos mensuales de dos especies de aves rapaces, el aguililla cola-roja Buteo jamaicensis y el cernícalo americano Falco sparverius. Los muestreos se realizaron en áreas naturales continuas y en áreas fragmentadas por actividad agrícola en el matorral desértico de Baja California Sur. En total se registraron en 1,806 ocasiones las dos especies. La más común fue el cernícalo americano con 1,164 (64.4\%) registros (los cuales fueron 51\% en área fragmentada y 49\% área natural) y el aguililla cola-roja con 642 registros (35.6\%) (70\% en área fragmentada y 30\% en área natural). Se estimaron las abundancias relativas y la densidad, las cuales no variaron durante la primavera y verano para el aguililla cola-roja, pero sus abundancias y densidades fueron mayores en las áreas fragmentadas durante otoño e invierno. Por otro lado, la abundancia del cernícalo americano fue similar entre áreas en todas las temporadas del año, mientras que la densidad fue similar en primavera, verano e invierno, pero fue mayor en el área fragmentada durante el otoño. Se encontró que el matorral desértico y sus áreas fragmentadas dentro de una matriz agrícola en Baja California Sur es un hábitat importante para las poblaciones migratorias de ambas especies de rapaces. Se discute sobre la tolerancia de las dos especies en función de su tamaño y selección de hábitat para anidar y forrajear, así como los efectos de la fragmentación del desierto sobre su abundancia y densidad en el sur de la península de Baja California.

Palabras clave: fragmentación, desierto, aguililla cola-roja, cernícalo americano, Baja California Sur, México, Buteo jamaicensis, Falco sparverius.

Tinajero, R. \& R. Rodríguez-Estrella. 2012. Effects of desert scrub habitat fragmentation on resident and migrant populations of Red-tailed Hawk and American Kestrel in Baja California Sur, Mexico. Acta Zoológica Mexicana (n. s.), 28(2): 427-446.

ABSTRACT. To evaluate the effects of desert scrub habitat fragmentation on resident and migrant populations of Red-tailed Hawk and American Kestrel, we conducted monthly raptor road surveys dur-

Recibido: 14/01/2012; aceptado: 24/02/2012. 
ing two years, 2008 and 2009, in natural desert scrub areas and in fragmented areas of Baja California Sur, México. We recorded 1,806 records of the two raptor species. The most common was the American Kestrel with 1,164 (64.4\%) records (51\% found in fragmented area, and 49\% in natural area). The Redtailed Hawk accounted for 642 records (35.6\%) (70\% in fragmented areas, and 30\% in natural areas). The relative abundance and density of Red-tailed Hawk in the spring and summer were similar between areas, but in the fall and winter the abundances and densities were much higher in fragmented areas. The abundance of American Kestrel was similar between areas in all seasons. Density was similar between areas in spring, summer and winter but it was higher in fragmented areas in the fall. The desert scrub of Baja California Sur and fragmented areas inside a cultivated agricultural matrix is an important habitat for migrant populations of both raptor species as indicated by the results of the fall and winter seasons. We discuss on the raptor species tolerance to habitat changes (threshold concept) as a function of their body size and habitat specialization (for nesting and foraging activities) and on the effects of fragmentation of desert scrub habitats on the abundance and density of the Red-tailed Hawk and American Kestrel in southern Baja California peninsula.

Key words: habitat fragmentation, desert scrub, Red-tailed Hawk, American Kestrel, Baja California Sur, México.

\section{INTRODUCCIÓN}

La fragmentación y pérdida de hábitat son dos de las principales amenazas a la biodiversidad que han provocado el decremento de poblaciones e inclusive extinciones locales (Turner 1996, Fahrig 1997, 2003). Distintas características de las especies las hacen proclives a ser negativamente afectadas por la actividad humana que produce pérdida del hábitat, cambios en el uso de la tierra y la fragmentación. Entre estas características se encuentra su demografía relacionada al tamaño poblacional, su grado de especialización al hábitat y su dieta (Siriwardena et al. 1998, Devictor et al. 2008, Davison \& Fitzpatrick 2010), pero también el grado de aislamiento que tengan dentro de un sistema fragmentado (Andrén 1994, Davies \& Margules 1998). No obstante, algunas especies pueden ser beneficiadas en algún grado por las actividades humanas (Bird et al. 1996, Rodríguez-Estrella et al. 1998, Filloy \& Bellocq 2007). Esto plantea un reto para la conservación de las especies pues sus estrategias y respuestas pueden diferir. Por ello es necesario entender las consecuencias que tienen las modificaciones al hábitat sobre la abundancia y distribución de las especies (frecuentemente de manera individual, especie por especie) con el fin de implementar mejores medidas de manejo, y conservación.

Los efectos de la fragmentación del hábitat sobre la distribución y abundancia de las especies han sido estudiados, aunque con limitaciones, en plantas, aves, mamíferos, reptiles e insectos para algunos ecosistemas del mundo, sobre todo en bosques tropicales y templados (Bierregaard et al. 1992, Robinson et at. 1992, Andrén 1994, Turner 1996, Davies \& Margules 1998, Laurance et al. 2000, Santos \& Tellería 2006). En aves, la mayoría de las investigaciones se han enfocado al grupo de paseriformes, encontrando en general efectos negativos sobre la riqueza y abundancia de las especies (Kattan et al. 1994, Knick \& Rotenberry 1995, Hobson \& Bayne 2000, 
Santos et al. 2002). Para las aves rapaces estos efectos son contrastantes, ya que para algunas especies este efecto es negativo, pero para otras puede beneficiarles. Las aves rapaces por su posición en las redes tróficas son consideradas como depredadores tope y su importancia radica en que determinan la estructura y dinámica de los ecosistemas (Sergio et al. 2005, 2008) y por ello es importante determinar los efectos de las actividades humanas sobre ellos, para entender la forma en que se modificará la funcionalidad en los ecosistemas. Los estudios sobre el efecto de la fragmentación en aves rapaces se han realizado básicamente en bosques tropicales y templados (Jullien \& Thiollay 1996, Bosakowsky \& Smith 1997). Sin embargo, la información es escasa o prácticamente inexistente para zonas desérticas (Pavez et al. 2010).

Estimar los tamaños de las poblaciones animales en su ambiente natural es importante porque nos dan información, entre otros, sobre las tendencias poblacionales y en un análisis temporal pueden mostrar cambios en la distribución y abundancia de una especie. Esta información es indispensable para realizar programas de conservación y manejo de especies (Southwood \& Henderson 2000). Dicha información nos permitirá comparar tamaños poblacionales de una especie dentro del mismo hábitat o con otras regiones o hábitats distintos, y en estaciones o años distintos, a distintas escalas. Los tamaños de poblaciones pueden ser afectados por factores tales como cambios en el número de animales en una etapa de desarrollo, cambios en su actividad ya que la actividad de los vertebrados terrestres por ejemplo, es ampliamente influenciada por cuestiones climáticas como la temperatura, precipitación, nubosidad, viento; cambios en la relación de sexos y otros datos por cohorte en la demografía de las especies, y por la eficiencia del método de muestreo (Southwood \& Henderson 2000). La información derivada de los muestreos de aves rapaces ha sido ya utilizada para estimar tamaños poblacionales y sus tendencias, localización de nidos, monitoreo del éxito reproductivo, distribución de especies, monitoreo de poblaciones de rapaces migratorias y para estudiar su ecología del comportamiento (Bird \& Bildstein 2007, Ruelas et al. 2010).

Los estudios realizados a la fecha en ecosistemas templados y tropicales fragmentados concluyen que el tamaño de los fragmentos o remanentes del hábitat natural condiciona la riqueza de especies rapaces y su abundancia (Thiollay 1993, Bosakowsky \& Smith 1997). Las aves rapaces tienen generalmente amplias áreas de caza ya que requieren de amplias extensiones de territorio para cazar sus presas y para llevar a cabo su reproducción (Newton 1979), por lo que ante un escenario de fragmentación del hábitat pueden ser consideradas como altamente vulnerables. Esta situación podría tener un similar o mayor impacto en las poblaciones de rapaces en zonas desérticas que en las templadas y tropicales. En un contexto general y siguiendo la idea de evaluar tendencias y efectos, consideramos importante determinar si los valores de los parámetros de abundancia y densidad se comportan de la misma manera en áreas naturales y en un área fragmentada de desierto que ha sufrido cambios por el hombre produciendo limitantes en disponibilidad de alimento y de sitios para anidar. 
Por otro lado, algunas investigaciones han encontrado que ciertas actividades humanas, como las agrícolas, pueden beneficiar a algunas especies por generarse "nichos” nuevos, tener una mayor disponibilidad de presas, y mayor visibilidad para la caza, lo cual es producto frecuentemente de una mayor heterogeneidad del paisaje (Valencia et al. 1980, Preston 1990, Donázar et al., 1993, Rodríguez-Estrella et al. 1998, Tella \& Forero 2000, Williams et al. 2000, Rodríguez- Estrella 2007, Cardador et al. 2011). El beneficio o afectación parecen relacionarse también al tamaño del área de caza y a los requerimientos del hábitat, los cuales dependen del tamaño de las especies (Giraudo et al. 2008).

En esta investigación nos centramos en realizar un análisis estacional de la presencia y abundancia de dos especies de aves rapaces en un área natural y dentro de un área fragmentada en el desierto de la península de Baja California. Lo anterior permitirá cuantificar los efectos de la fragmentación en la abundancia de ambas especies, tanto para las poblaciones residentes como para las migratorias. Las especies estudiadas fueron el aguililla cola-roja (Buteo jamaicensis) y el cernícalo americano (Falco sparverius), las que presentan una amplia distribución continental y utilizan una gran variedad de ambientes. Ambas especies están consideradas entre las rapaces más abundantes de Norteamérica (Preston \& Beane 1993). En el estado de Baja California Sur estas especies presentan poblaciones residentes y migratorias (RodríguezEstrella et al. 1998). Al seleccionar estas especies se quería probar si las condiciones del hábitat (natural vs. fragmentado) afectan de manera similar a especies de tamaño diferente, con dieta distinta y requerimientos de hábitat distinto. Este conocimiento nos permitirá identificar variables intrínsecas y extrínsecas que afectan a las especies, lo cual nos permitirá hacer mejores propuestas de manejo de las especies en el sistema de desierto. El aguililla cola-roja es de tamaño mediano, teniendo entre 45 y $65 \mathrm{~cm}$ de largo y con un peso oscilando entre 690 y $1460 \mathrm{~g}$. Se distribuye desde Alaska, pasando por Canadá, Estados Unidos de Norteamérica, México y hasta Panamá (Preston \& Beane 1993). Para anidar, utiliza generalmente acantilados, en ocasiones árboles grandes y cactáceas columnares altas en áreas desérticas, tales como saguaros (Preston \& Beane 1993, Baicich \& Harrison 2005). Las poblaciones de esta especie en Norteamérica, en su área reproductiva, permanecen estables e inclusive se incrementan, pero sus poblaciones migratorias en México han mostrado un descenso (Ruelas et al. 2010). El cernícalo americano es el halcón más pequeño de Norteamérica, mide entre 22 y $31 \mathrm{~cm}$ y pesa entre 80 y 165 gr. Presenta una distribución muy amplia en el continente americano. Para anidar, utiliza cavidades naturales en árboles así como cavidades construidas por pájaros carpinteros, huecos en construcciones, huecos en rocas y en bancos de tierra (Baicich \& Harrison 2005). Recientemente se ha documentado que sus poblaciones en Norteamérica, tanto reproductoras como migratorias, están disminuyendo (Sullivan \& Wood 2005, Sauer et al. 2008, Farmer \& Smith 2009, Smallwood et al 2009, Ruelas et al. 2010). Entre las causas principales 
de su disminución se sugiere la pérdida del hábitat. Aunque aún existe controversia de cuál es la verdadera causa de su disminución, se ha sugerido que probablemente el problema se encuentre en las áreas donde sus poblaciones pasan la temporada de migración o a lo largo de sus rutas migratorias (Farmer \& Smith 2009, Smallwood et al. 2009).

En resumen, la presente investigación evalúa los cambios estacionales y anuales que se presentan en la abundancia de estas dos especies que usaremos como modelo, que tienen poblaciones residentes y migratorias en el área de estudio, así como también hace una comparación entre las abundancias relativas y densidades de estas dos especies con tamaños corporales y requerimientos de hábitat y dieta distintos en cuanto a su área de caza. Para ello, se evalúan estos parámetros en un área natural continua con un nivel de disturbio humano bajo y en un área fragmentada con un nivel de disturbio alto. Con esta información podremos evaluar el efecto que ha tenido la fragmentación del hábitat del matorral desértico sobre las abundancias de las poblaciones reproductoras y migratorias de las especies indicadas, así como también permitirá evaluar si las áreas fragmentadas rodeadas de una matriz agrícola permiten que las poblaciones de estas rapaces puedan permanecer para realizar su reproducción y para pasar el invierno.

\section{MATERIAL Y MÉTODOS}

El estudio se realizó en la parte central del estado de Baja California Sur, en el municipio de Comondú, en el área conocida como Valle de Santo Domingo (Figura 1). Este valle tiene una extensión de $2200 \mathrm{~km}^{2}$ con 67,200 hectáreas dedicadas a la agricultura (Salinas-Zavala et al. 2006). El clima que predomina es del tipo Bw, clima muy seco, con subtipos muy secos y muy cálidos (INEGI 1995). La precipitación media anual es de $158.2 \mathrm{~mm}$ anuales (Corral et al. 2006). La vegetación en el área de estudio es matorral desértico. Se caracteriza por la presencia de especies arbustivas y tallos carnosos. Las plantas predominantes en la zona natural son los torotes (Bursera spp.), mezquites (Prosopis spp.), palo Adán (Fouquieria diguetii), palo verde (Cercidium microphylum), palo blanco (Lysiloma candidum), lomboy (Jatropha cinerea), choya (Opuntia choya), cardón (Pachycereus pringleii), pitaya agria (Stenocereus gummosus), pitaya dulce (Lemaireocereus turberi)(Wiggins 1980). La agricultura de riego que se ha practicado en el Valle de Santo Domingo inició formalmente entre los años de 1950-1960 cuando el gobierno federal impulsó la colonización de esta región (Tejas et al. 1991). En años recientes debido a un uso desmedido de agua de riego, ha disminuido la superficie agrícola, los rendimientos por hectárea y la calidad del suelo. Desde 1950, con el comienzo de la agricultura y con el cambio de uso del suelo se ha presentado un proceso gradual de fragmentación del matorral desértico en esta área, incrementándose a partir de 1980 (Rodríguez-Estrella et al. datos no publ.). El área 


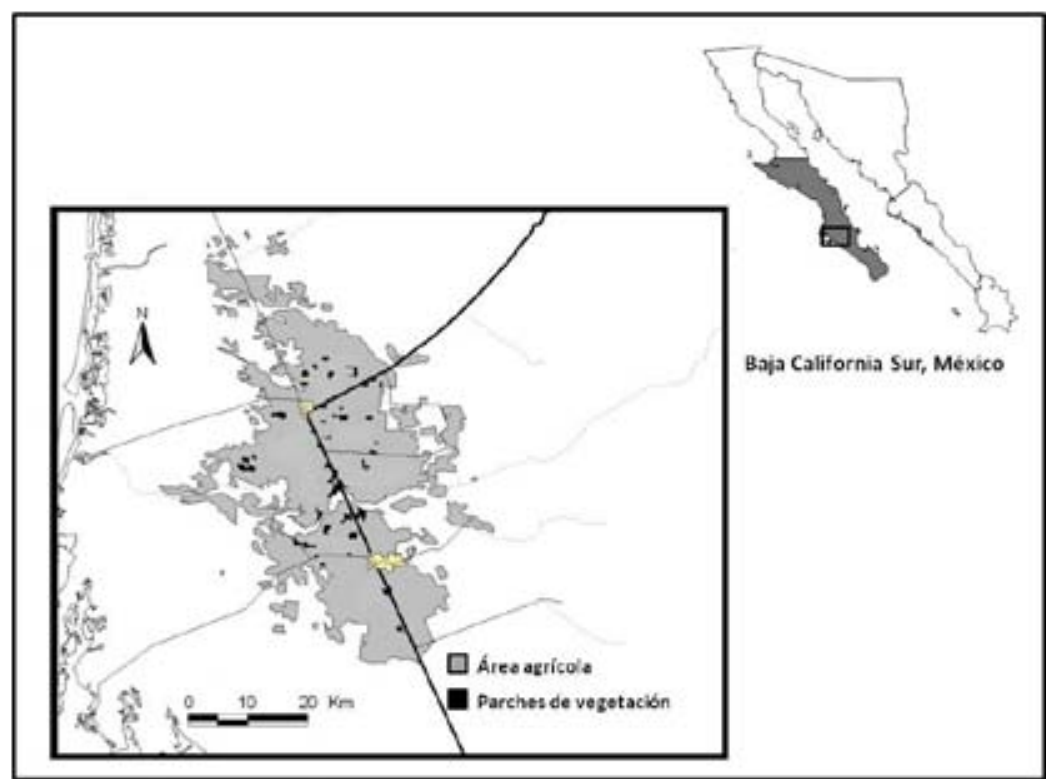

Figura 1. Localización de área de estudio en el estado de Baja California Sur.

fragmentada donde realizamos el estudio consta de más de 100 parches de vegetación natural de distintos tamaños, de entre 1 a 954 hectáreas, que se encuentran inmersos en una matriz agrícola.

Estimación de abundancia relativa y densidad. El muestreo de las aves rapaces se realizó utilizando una metodología estándar que consiste en establecer transectos a lo largo de caminos secundarios, recorriendo una distancia conocida en vehículo a una velocidad promedio de $30 \mathrm{~km} / \mathrm{h}$. Dos personas registraron todos los individuos observados en una banda de ancho fijo de $200 \mathrm{~m}$ a cada lado del camino (Fuller \& Mosher 1987). Las abundancias relativas se consideraron como el número de aves registradas por la longitud del transecto, valor que posteriormente se estima en aves $/ 100 \mathrm{~km}$. Posteriormente, los valores fueron agrupados y promediados por mes y por estación del año. Esta medida (aves/100 km) permite hacer comparaciones de abundancia de las rapaces en distintas temporadas así como con otros estudios.

La densidad de las aves fue obtenida de los datos de los muestreos considerando la distancia perpendicular al transecto de cada observación. Los datos fueron agrupados por estación, área y año, y se utilizó el programa DISTANCE. Este programa corrige el decremento de detectabilidad de las aves según aumenta la distancia respecto al centro del transecto; basándose en este decremento estima cuál es la densidad "real" de la especie muestreada. La fórmula utilizada es $D=(n f(0)) / 2 L$, donde $D$ es la den- 
sidad, (n) el número total de aves detectadas, $f(0)$ la función de detección estimada por el programa, y L es la distancia lineal total muestreada (Thomas et al. 2010). Para obtener distancias precisas, se realizaron pruebas de estimación de distancia utilizando un equipo Range-Finder (Opti-Meter, Ranging 620).

Estos métodos de muestreo y estimación de abundancias han sido ampliamente utilizados en diferentes ecosistemas del mundo (Donázar et al. 1993, Williams et al. 2000, Seavy \& Apodaca 2002, Ferguson 2004, Golstein \& Hibbitts 2004, Carrete et al. 2009), y tienen la ventaja de que permiten hacer comparaciones posteriores. La identificación de las aves se realizó utilizando binoculares 8 X40 y guías de campo de aves (Sibley 2000, Clark \& Wheeler 2001).

Ubicación de transectos. Cuatro transectos de $27 \mathrm{~km}$ cada uno fueron establecidos, dos en áreas naturales y dos en el área fragmentada. El área natural se denomina aquella donde la vegetación natural dominante de la región se conserva en grandes extensiones de superficie y la actividad humana se restringe a la existencia de ranchos pequeños aislados separados entre ellos varios kilómetros, donde existe un nivel de disturbio humano bajo. El área fragmentada es aquella donde la actividad agrícola restringió la ubicación de la vegetación natural en una disposición de parches aislados (conteniendo más de 100 parches de vegetación natural de distintos tamaños), que se encuentran inmersos en una matriz agrícola con un nivel de disturbio alto.

Los transectos de cada tipo fueron separados por una distancia mínima de 17 km teniendo así dos repeticiones, uno de otro en su tipo. Los transectos fueron recorridos dos veces al mes durante 2008 (enero a diciembre) y durante nueve meses en 2009 (excepto abril, junio y agosto). Los transectos se recorrieron durante la mañana (0700-1000 h), iniciando una hora después de la salida del sol, y en la tarde, tres horas antes de la puesta del sol (1600-2000 h). En estos horarios se incrementa la detectabilidad de las aves rapaces.

Análisis de datos. Para determinar si las abundancias de las especies fueron diferentes estacionalmente en diferentes condiciones del hábitat (natural y fragmentado), se hizo un análisis de varianza de muestras repetidas, con la opción para modelos lineales generalizados (GLM), utilizando el programa SPSS, versión 11.5. La abundancia de aves por transecto o los "transectos" fueron definidos como los sujetos, la estación (primavera, verano, otoño e invierno) fue el factor intra-sujetos, y el área o condición del hábitat, la variable inter-sujetos. El ANOVA de muestras repetidas es utilizado cuando se realizan múltiples observaciones sobre la misma unidad experimental (Crowder \& Hand 1990), en este caso "el transecto". Este análisis permite contrastar los efectos de diferentes estaciones del año y también contrastar en cada año en cada nivel de la variable (área) dentro de cada nivel del factor (estación).

Para comparar los datos de densidad promedio de las aves rapaces entre diferentes áreas, se aplicó la prueba t-student (Zar 1996). 


\section{RESULTADOS}

Registros. Durante los dos años de muestreo se recorrieron un total de $4472 \mathrm{~km}$, registrando en 642 ocasiones el aguililla cola-roja, con 70\% de los registros en áreas fragmentadas y el 30\% en áreas naturales (Cuadro 1). La mayoría de los registros ocurrió en la estación de otoño en ambas áreas de muestreo, con 50.9\% en áreas fragmentadas y $50 \%$ en áreas naturales. La primavera fue la estación en que se registró el menor número de individuos, siendo de $<3 \%$ en ambas áreas (Cuadro1). El cernícalo americano fue registrado en 1164 ocasiones, de las que 51\% fueron en áreas fragmentadas y $49 \%$ en áreas naturales (Cuadro 1). Otoño fue la estación con mayor número de registros del cernícalo americano, con $48.7 \%$ en áreas naturales y $47.1 \%$ en áreas fragmentadas. La primavera presentó menos del 9\% de los registros en ambas áreas (Cuadro 1).

Abundancia relativa. La abundancia del aguililla cola-roja entre los transectos de las áreas fragmentadas no mostró diferencias estacionales. La abundancia fue significativamente diferente entre los transectos de áreas naturales en primavera-verano $(\mathrm{F}=4.76$, g.l.:1,22, $\mathrm{p}=0.04)$ e invierno ( $\mathrm{F}=4.35$, g.l.:1,22, $\mathrm{p}=0.049)$. Por otro lado, las abundancias del cernícalo americano no fueron significativamente diferentes entre los transectos del área fragmentada en las estaciones del año, pero sí hubo diferencias en las abundancias en los transectos del área natural en invierno ( $F=13.6$, g.l.:1,22, $\mathrm{p}=0.001$ ).

Cuadro 1. Número de registros de Buteo jamaicensis y Falco sparverius en dos áreas (natural y fragmentada) en cuatro estaciones de los años 2008-2009. Se presenta el esfuerzo de muestro realizado en los dos años.

\begin{tabular}{|c|c|c|c|c|c|c|c|c|c|}
\hline \multirow{3}{*}{ Área Natural } & \multicolumn{2}{|c|}{ Primavera } & \multicolumn{2}{|c|}{ Verano } & \multicolumn{2}{|c|}{ Otoño } & \multicolumn{2}{|c|}{ Invierno } & \multirow[t]{2}{*}{ Total } \\
\hline & \multicolumn{8}{|c|}{ Distancia recorrida } & \\
\hline & $432 \mathrm{~km}$ & & $540 \mathrm{~km}$ & & $648 \mathrm{~km}$ & & $648 \mathrm{~km}$ & & $2268 \mathrm{~km}$ \\
\hline Área Fragmentada & $432 \mathrm{~km}$ & & $486 \mathrm{~km}$ & & $648 \mathrm{~km}$ & & $648 \mathrm{~km}$ & & $2214 \mathrm{~km}$ \\
\hline Buteo jamaicensis & & $(\%)$ & & $(\%)$ & & $(\%)$ & & $(\%)$ & \\
\hline Área natural & 5 & 2.6 & 12 & 6.3 & 95 & 50 & 78 & 41.1 & 190 \\
\hline Área fragmentada & 3 & 0.7 & 9 & 2 & 230 & 50.9 & 210 & 46.5 & 452 \\
\hline Total & 8 & & 21 & & 325 & & 288 & & 642 \\
\hline \multicolumn{10}{|l|}{ Falco sparverius } \\
\hline Área natural & 48 & 8.5 & 78 & 13.8 & 275 & 48.7 & 164 & 29 & 565 \\
\hline Área fragmentada & 38 & 5.8 & 109 & 18.2 & 282 & 47.1 & 170 & 28.4 & 599 \\
\hline Total & 86 & & 187 & & 557 & & 334 & & 1164 \\
\hline Gran total & 94 & & 208 & & 882 & & 622 & & 1806 \\
\hline
\end{tabular}


Las abundancias relativas por temporada del aguililla cola-roja en el área natural no fueron significativamente diferentes entre años (Apéndice 1, figura 2). En el área fragmentada las abundancias no fueron estadísticamente diferentes entre años, pero sí se encontraron diferencias al comparar invierno entre años: la abundancia relativa en 2008 fue mayor con 43.8 aves/100km y menor en 2009 con 20.99 aves/100km (F $=5.48$, g.l.:1, 22, $\mathrm{p}=0.03$ ).

En área natural el cernícalo americano mostró un patrón distinto entre años. La abundancia en primavera-verano de 2008 (10.2 aves/100 km) fue menor que la registrada en 2009 (18.5 aves/100 km) (Apéndice 1; $\mathrm{F}=5.42$, g.l.: l, 22, $\mathrm{p}=0.03$ ). La abundancia en el otoño de 2008 fue significativamente menor que en 2009 (Apéndice $1 ; \mathrm{F}=11$, g.l.:1, 22, $\mathrm{p}=0.003$ ). La abundancia en invierno fue similar entre años (Apéndice 1; $\mathrm{F}=0.006$, g.l.:1, 22, $\mathrm{p}=0.94$ ). En el área fragmentada la abundancia
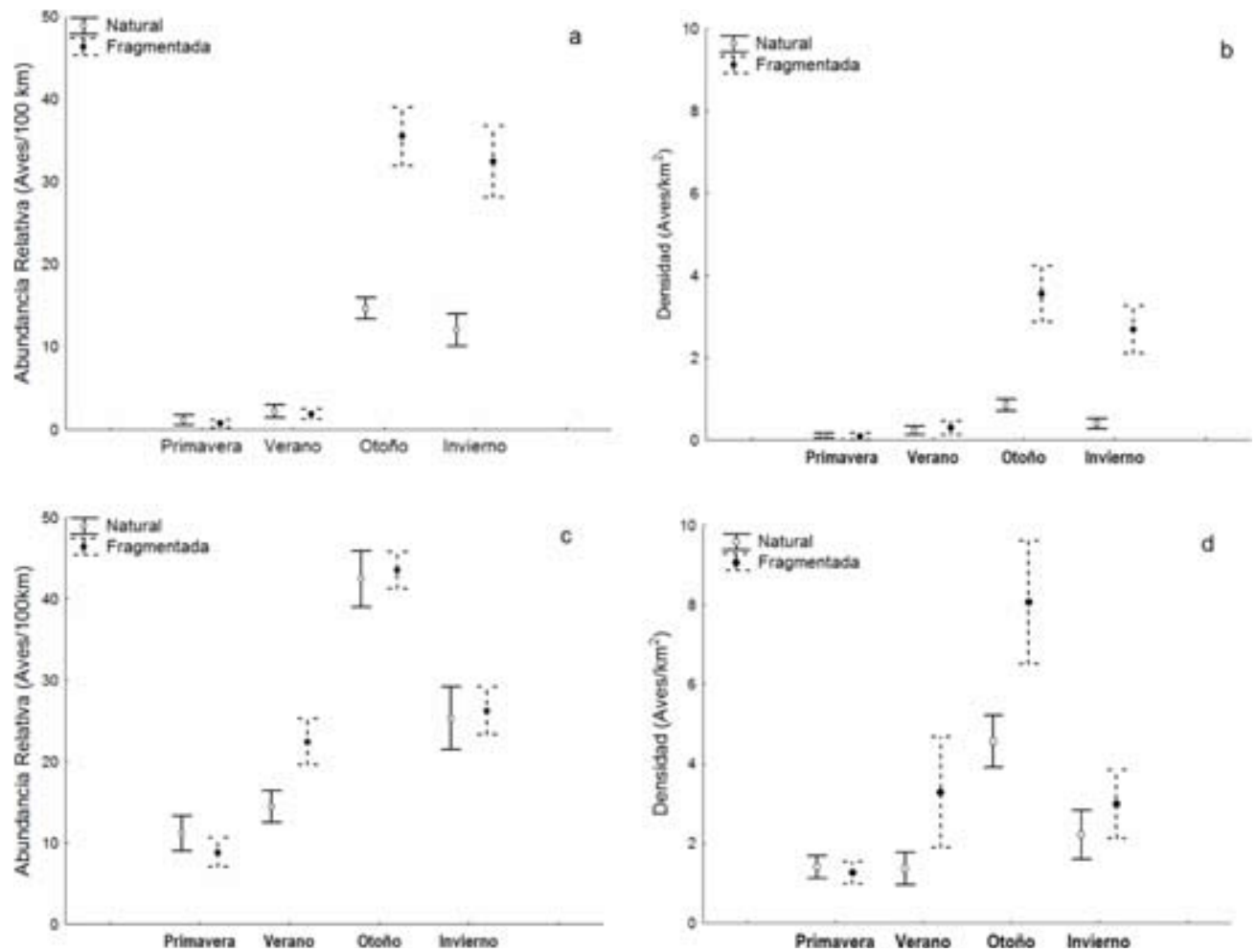

Figura 2. (a) Abundancia relativa de la aguililla cola-roja por área y por estación (aves/100km). (b) Densidad del aguililla cola-roja por área y estación (aves $/ \mathrm{km}^{2}$ ). (c) Abundancia relativa del cernícalo americano por área y por estación (aves/100km). (d) Densidad del cernícalo americano por área y estación (aves $/ \mathrm{km}^{2}$ ). 
únicamente fue diferente entre años en la estación de otoño (34.5 aves/100 km en 2008, 52.5 aves/100 km en 2009; Apéndice 1; $\mathrm{F}=31.35$, g.l.:1, 22, $\mathrm{p}<0.0001$ ).

\section{Abundancia estacional en función del tipo de ambiente, Fragmentado vs. Na-}

tural. No se encontraron diferencias en la abundancia del aguililla cola-roja entre el área fragmentada y la natural ni en 2008 ni en 2009 en la temporada primavera-verano, pero sí en áreas fragmentadas para otoño $\left(\mathrm{F}_{2008}=33\right.$, g.l.:1, 22, $\mathrm{p}<0.0001 ; \mathrm{F}_{2009}=$ 10.9, g.l.:1, 22, $\mathrm{p}=0.003)$ e invierno $\left(\mathrm{F}_{2008}=11.2\right.$, g.l.:1, 22, $\mathrm{p}=0.003 ; \mathrm{F}_{2009}=5.57$, g.l.:1, 22, $\mathrm{p}=0.03$ ) (Apéndice 2 y figura 2). Para el cernícalo americano, las abundancias relativas registradas para todas las estaciones del año y entre años no fueron significativamente diferentes (Apéndice 2 y figura 2).

Para el aguililla cola-roja las abundancias fueron similares entre áreas en la temporada de primavera-verano ( $\mathrm{F}=0.4$, g.l.:1, $46, \mathrm{p}=0.53$ ) mientras que en otoño e invierno las diferencias fueron altamente significativas, observando mayores abundancias en las áreas fragmentadas en otoño $(\mathrm{F}=27.26$, g.l.:1,46, $\mathrm{p}<0.0001)$ y en invierno ( $\mathrm{F}=14.47$, g.l.:1, 46, $\mathrm{p}<0.0001$ ) (Cuadro 2). Las abundancias relativas del cernícalo americano fueron similares en los dos años de estudio, no se encontraron diferencias en las abundancias para las diferentes estaciones del año (Cuadro 2).

Densidad. La densidad del aguililla cola-roja fue similar entre ambas áreas en las estaciones de primavera-verano (Cuadro 3; $\mathrm{t}=0.22$, g.l.: $8, \mathrm{p}=0.828$ ). En el otoño e invierno la densidad fue significativamente mayor en las áreas fragmentadas ( $\mathrm{t}_{\text {otoño }}$ $=3.72$, g.l.:5, $\mathrm{p}=0.014$; $\mathrm{t}_{\text {invierno }}=4.64$, g.l.:5, $\mathrm{p}=0.006$ ). La densidad del cernícalo americano fue similar en el área fragmentada y natural en primavera-verano (Cuadro 3 ; $\mathrm{t}=1.52$, g.l.:8, $\mathrm{p}=0.16)$ y en invierno $(\mathrm{t}=1.03$, g.l.:5, $\mathrm{p}=0.35)$. En las estaciones de otoño las densidades fueron distintas, con $4.56 \pm 0.69$ aves $/ \mathrm{km}^{2}$ en el área natural

Cuadro 2. Valores promedio de abundancia ( $\underline{\underline{ \pm} \text { e.s. }}$ ) juntando los datos registrados en 2008-2009.

Se hacen las comparaciones considerando el valor de aves/100 km. En negritas están los valores que dieron significativos en las comparaciones.

\begin{tabular}{lcccc}
\hline & Área Natural & Área Fragmentada & $\mathrm{f}$ & $(\mathrm{p})$ \\
\hline Primavera-Verano & & & & \\
Buteo jamaicensis & $1.75 \pm 0.54$ & $1.31 \pm 0.41$ & 0.4 & 0.53 \\
Falco sparverius & $12.96 \pm 1.5$ & $16.01 \pm 2.14$ & 0.82 & 0.37 \\
Otoño & & & $\mathbf{2 7 . 2 6}$ & $<\mathbf{0 . 0 0 0 1}$ \\
Buteo jamaicensis & $\mathbf{1 4 . 6 6} \pm \mathbf{1 . 4}$ & $\mathbf{3 5 . 4 9} \pm \mathbf{3 . 8}$ & 0.06 & 0.81 \\
Falco sparverius & $42.44 \pm 3.6$ & $43.52 \pm 2.4$ & & $\mathbf{0 . 0 0 0 1}$ \\
Invierno & & & $\mathbf{1 4 . 4 7}$ & 0.97 \\
Buteo jamaicensis & $\mathbf{1 2 . 0 4} \pm \mathbf{2 . 1}$ & $\mathbf{3 2 . 4 1} \pm \mathbf{4 . 5}$ & 0.001 & \\
Falco sparverius & $25.31 \pm 4.1$ & $26.23 \pm 3.12$ & & \\
\hline
\end{tabular}


Cuadro 3. Valores promedio de densidad ( \pm e.s.) juntando los datos registrados en 2008-2009. Se hacen las comparaciones considerando el valor de aves $/ \mathrm{km}^{2}$. En negritas están los valores que dieron significativos en las comparaciones.

\begin{tabular}{lcccc}
\hline & Área Natural & Área Fragmentada & $\mathrm{t}$ & $(\mathrm{p})$ \\
\hline Primavera-Verano & & & & \\
Buteo jamaicensis & $0.17 \pm 0.06$ & $0.20 \pm 0.10$ & 0.22 & 0.828 \\
Falco sparverius & $1.38 \pm 0.25$ & $2.39 \pm 0.86$ & 1.52 & 0.165 \\
Otoño & & & \\
Buteo jamaicensis & $\mathbf{0 . 8 4} \pm \mathbf{0 . 1 4}$ & $\mathbf{3 . 5 5} \pm \mathbf{0 . 7 2}$ & 3.72 & $\mathbf{0 . 0 1 4}$ \\
Falco sparverius & $4.56 \pm 0.69$ & $8.06 \pm 1.63$ & 3.27 & 0.022 \\
Invierno & & & & \\
Buteo jamaicensis & $\mathbf{0 . 3 9} \pm \mathbf{0 . 1 2}$ & $\mathbf{2 . 6 8} \pm \mathbf{0 . 6 1}$ & $\mathbf{3 . 6 8}$ & $\mathbf{0 . 0 0 6}$ \\
Falco sparverius & $2.22 \pm 0.64$ & $2.99 \pm 0.91$ & 1.04 & 0.347 \\
\hline
\end{tabular}

y de $8.06 \pm 1.63$ aves $/ \mathrm{km}^{2}$ en el área fragmentada, siendo de prácticamente el doble que en las áreas naturales ( $\mathrm{t}=3.27$, g.l.:5, $\mathrm{p}=0.02)$.

\section{DISCUSIÓN}

Se ha reportado que la riqueza y abundancia de aves rapaces disminuye con la fragmentación del hábitat (Chamberlain et al. 2000, Carrete et al. 2009, Butet et al. 2010). Sin embargo, en nuestro estudio no encontramos un aparente efecto negativo en la abundancia del aguililla cola-roja y cernícalo americano con relación a la fragmentación del desierto. Pero sí hubo variación en los índices de abundancia en las distintas temporadas del año y entre años para algunas temporadas. La variación en las abundancias entre 2008 y 2009 para el aguililla cola-roja y el cernícalo americano en el otoño e invierno se debe al incremento en el número de individuos seguramente migratorios durante esta temporada, o bien a las variaciones en productividad de las especies o a variaciones ambientales que inducen a que los individuos de ambas rapaces lleguen a pasar el invierno a la península. Puede deberse también a cambios interanuales de las poblaciones de rapaces que se relacionen con los patrones climáticos regionales, como eventos de El Niño, o con incrementos en la temperatura invernal en un año determinado (ver Kim et al. 2008, Ruelas et al. 2010), lo que debe modificar la disponibilidad de alimento, principalmente.

Las diferencias en abundancias relativas de las especies entre temporadas y anuales, se deben básicamente a las poblaciones residentes en primavera-verano (cuando se presentan valores menores), ya que es la época reproductiva, mientras que en el otoño-invierno aparecen los individuos migratorios que se suman a los individuos residentes de las poblaciones estudiadas. Nuestros resultados coinciden con lo reporta- 
do anteriormente para ambas especies en el sur de la península de Baja California ya que durante las estaciones de primavera-verano no se encontraron variaciones en la abundancia del aguililla cola-roja y del cernícalo americano en zonas humanizadas y naturales (Rodríguez-Estrella et al. 1998). De esta manera, en nuestro estudio parece que la fragmentación del hábitat no tiene un efecto sobre la abundancia de las poblaciones residentes de estas dos especies de rapaces. No obstante, se ha determinado también que en la época reproductiva se presentan menos individuos del aguililla cola-roja en las zonas cercanas a campos agrícolas y en zona fragmentada en relación a la zona natural, mientras que el cernícalo incrementa sus números en zonas agrícolas, aparentemente siendo beneficiado por estas actividades (Rodríguez-Estrella 2007). Estas tendencias parecen explicarse porque el aguililla cola-roja anida principalmente en acantilados y zonas de cerros en Baja California Sur, aunque puede muy ocasionalmente anidar en grandes cardones (Rodríguez-Estrella et al. datos no publ.). No hemos registrado ni un solo nido del aguililla cola-roja en la zona fragmentada en más de 7 años de estudios en la zona. El cernícalo por el contrario puede tener nidos tanto en la zona natural como en la fragmentada y se alimenta frecuentemente en los bordes de los parches.

Por otro lado, si comparamos nuestros resultados con lo observado para especies ecológicamente similares como el ratonero común (Buteo buteo) y el cernícalo vulgar (Falco tinnunculus) en áreas agrícolas de Francia, los resultados difieren ya que la abundancia de estas dos especies presentó una tendencia negativa con la fragmentación y pérdida de hábitat al reducirse las arboledas (hedgerows), los bosques y las áreas de pastizal en esa región. Aunque el cernícalo vulgar fue menos afectado por las modificaciones humanas del paisaje (Butet et al. 2010). En España, se encontró que el cernícalo vulgar era más abundante en las áreas con mayor cobertura de pastizal, y además en terrenos con mayor cobertura de cultivos de temporal que no requieren irrigación (Campion 2004). En este sentido, al parecer las rapaces del género Falco de tamaño pequeño y que colocan sus nidos en cavidades, son más tolerantes a las modificaciones del hábitat natural, e inclusive parecen beneficiarse de la apertura del hábitat natural ya que aparentemente pueden cazar más fácilmente en estas zonas abiertas (McCrary et al. 1985, Hoffman \& Smith 2003, Jensen et al. 2005). Los halcones del género Buteo construyen nidos más elaborados y requieren de sitios más complejos, tales como los acantilados o cortados en paredes de cerros y montañas. En la zona de estudio del valle de Santo Domingo esta condición no existe. Las montañas más cercanas se encuentran a más de 40 kilómetros hacia la sierra, donde es factible que los individuos reproductores residentes del aguililla cola-roja migren localmente para reproducirse.

Al analizar la temporada de otoño e invierno se encontró que los índices de abundancia variaron para las dos especies. El aguililla cola-roja presentó mayores abundancias en las áreas fragmentadas que en las áreas naturales, mientras que las 
abundancias del cernícalo americano no variaron entre áreas. Contrario a lo encontrado en este estudio en cuanto al efecto de los cambios de uso de suelo, en la porción sur de Baja California Sur las abundancias del aguililla cola-roja durante el invierno entre áreas agrícolas y naturales no fueron diferentes, pero sí coincidió con lo encontrado para el cernícalo americano pues se reportó una mayor abundancia en áreas agrícolas que en áreas naturales (Rodríguez-Estrella et al. 1998). Es decir, en la parte sur de la península las poblaciones del aguililla cola-roja durante el invierno parecen distribuirse ampliamente para usar diferentes ambientes. Se ha encontrado en especies de aves que durante la época de reproducción cuando solo hay poblaciones residentes, los individuos forrajean en todos los hábitats disponibles, aunque se encuentran más frecuentemente en aquellos que prefieren. Pero en la temporada de otoño-invierno con la llegada de individuos migratorios, las residentes parecen concentrarse en los hábitats preferidos mientras que los individuos migratorios utilizan todos los ambientes, segregándose de alguna manera (Kirk \& Currall 1994). Además con la llegada de los individuos migratorios que son de talla más grande y probablemente más agresivos y dominantes que los residentes, éstos últimos pueden ser desplazados por lo que se concentran solo en algunos sitios preferidos (Kirk \& Gosler 1994). De esta manera, las poblaciones residentes del aguililla cola-roja en la parte sur de la península de Baja California donde hay menos sitios abiertos para la agricultura, utilizarían la vegetación natural que es la que generalmente prefieren (Rodríguez-Estrella et al. 1998, Rodríguez-Estrella 2007), en tanto los individuos de poblaciones migratorias utilizarían además las zonas agrícolas, más abiertas. En el valle agrícola de la mitad del estado de Baja California Sur, proponemos que son los individuos migratorios quienes principalmente utilizan las zonas fragmentadas y agrícolas, mientras que los individuos de las poblaciones residentes utilizan la vegetación natural como las zonas montañosas alejadas del valle (Rodríguez-Estrella 2007).

Las abundancias estimadas en este estudio para el aguililla cola-roja en las áreas fragmentadas ( 35.5 aves/100 km) y en áreas naturales (14.66 aves/100 km) en otoño-invierno fueron menores a lo encontrado en el invierno en un área agrícola en California, EUA (50 individuos/100 km; McCrary et al. 1985), pero mayor o similar a lo encontrado en el invierno en el desierto Sonorense (19 individuos/100 km; Marin \& Schmitt 1996). Para el cernícalo americano sus abundancias en el otoño (43.52 aves/100 km en área fragmentada y 42.44 aves/100 km en área natural), fueron menores a las reportadas en invierno para áreas agrícolas de California (70 aves/100 km; McCrary et al. 1985). Por el contrario, sus abundancias fueron muy superiores a las encontradas en el desierto de Sonora (10 aves/100 km; Marin \& Schmitt 1996). Lo anterior sugiere que dentro de la región Noroeste de México, la península de Baja California es aparentemente un sitio importante para la migración de cernícalos que prefieren las áreas abiertas, fragmentadas, dentro de una matriz agrícola. 
En general, se han usado estimaciones de abundancias relativas para determinar las variaciones de rapaces a través de largos transectos y regiones (McCrary et al. 1985, Donazar et al. 1993, Lish \& Burge 1995, Goldstein \& Hibbitts 2004, Jensen et al. 2005, Carrete et al. 2009). Pocos trabajos han reportado densidades (Andersen et al. 1985, Thiollay 1989, Boano \& Toffoli 2002, Nikolov et al. 2006). Consideramos que es importante presentar información tanto de abundancias relativas como de densidad para hacer mejores comparaciones. La densidad puede evaluar el número de individuos por unidad de área, siendo esta una mejor estimación constante y de variación dentro de áreas o regiones, que la abundancia relativa que no indica agrupaciones. En muestreos de campo, la detectabilidad de las aves variará por diversos factores, uno de ellos el tamaño de las especies (Goldstein \& Hibbitts 2004). En nuestro estudio cuando comparamos la abundancia y densidad del aguililla cola-roja (especie de mayor tamaño corporal) la tendencia en su abundancia y densidad resulta ser muy similar. Sin embargo, en el caso del cernícalo americano (especie de pequeño tamaño corporal) no encontramos diferencias en otoño al comparar las abundancias, pero sí se encontraron diferencias significativas al comparar la densidad, presentado mayor densidad en áreas fragmentadas. Esto puede cambiar la interpretación de los resultados si no se tiene cuidado o mayor información. Con lo anterior recomendamos que se usen las estimaciones tanto de abundancia como de densidad al hacer comparaciones de las poblaciones de rapaces, sobre todo para especies de tamaño pequeño.

Es importante considerar que el grado de fragmentación y la pérdida de hábitat pueden condicionar la presencia y abundancia de aves rapaces, ya que aunque pudieran tener abundancias similares entre zonas naturales y fragmentadas, e incluso ser mayores en las fragmentadas, llegado el umbral de tolerancia ("threshold") en cuanto a la cantidad de hábitat adecuado disponible, las poblaciones pueden decrecer dramáticamente (Kattan et al. 1994, Campion 2004, Pavez et al. 2010). En nuestro estudio, no se detectó un efecto negativo, de decremento de las poblaciones residentes y migratorias del cernícalo americano en zonas fragmentadas, pero se esperaría que si se incrementa el grado de fragmentación teniendo una mayor proporción de parches pequeños, sus abundancias o poblaciones podrían disminuir rápidamente (ver Filloy \& Bellocq 2007, Butet et al. 2010, Pavez et al. 2010). También es importante considerar que se debe ser cuidadoso con los datos de densidad ya que no siempre el tener altas densidades en un área significa una buena calidad de hábitat, considerando que especies generalistas pueden tener una alta densidad pero su población estar constituida por individuos inmigrantes en su mayoría y en estas áreas el éxito reproductivo de las residentes puede ser bajo (Van Horne 1983).

La fragmentación puede afectar de manera diferente a cada especie. Por ejemplo, en Uganda, África se encontró una mayor abundancia de aves rapaces en áreas agrícolas, pero las especies especialistas de bosque fueron poco frecuentes (Seavy \& Apodaca 2002). En la India, en los bosques tropicales, se encontró que especies que 
presentan tolerancia a la fragmentación fueron Elanus caeruleus y Falco tinnunculus, que se asocian a hábitats abiertos tales como pastizales y áreas de cultivo; sin embargo, la distribución de especies más especialistas se restringió únicamente a bosques tropicales. En Argentina, encontraron una mayor abundancia de aves rapaces en áreas agrícolas comparado con lo encontrado en el matorral desértico, y las especies más abundantes en áreas agrícolas fueron aquellas con mayor tolerancia a actividades humanas, tales como el chimango caracara (Milvago chimango) y el caracara ( $\mathrm{Ca}$ racara plancus) (Goldstein \& Hibbitts 2004). En la selva paranaense de Argentina y Paraguay las dos especies de rapaces que incrementaron sus abundancias (Falco sparverius y Athene cunicularia) se conocen por ser tolerantes a hábitats abiertos, mientras que las especies que disminuyeron sus abundancias, como el halcón montés (Micrastur spp.) y el milano plomizo (Ictinia plumbea) son más especialistas de hábitats boscosos (Zurita y Bellocq 2007). El grado de tolerancia de las especies a hábitats abiertos y zonas agrícolas es un factor que les permite usar ambientes que se van degradando. Las especies especialistas son las primeras en ser afectadas y desaparecer en estos ambientes, ya que presentan un menor umbral de tolerancia.

Más estudios se requieren para entender la manera en que las poblaciones residentes y migratorias de Buteo jamaicensis y Falco sparverius cambian por la apertura de áreas a cultivo y por la fragmentación. Pero podemos concluir que las estimaciones de abundancia y densidad en este estudio no mostraron un efecto claro de la fragmentación en estas dos especies de rapaces. Sin embargo, las poblaciones migratorias de ambas especies parecen usar más las zonas fragmentadas y de cultivos que las residentes en la porción sur de la península de Baja California. El uso de áreas fragmentadas y de cultivo en Baja California Sur y probablemente en el resto de México puede afectar a las poblaciones de aves, en particular a las migratorias de invierno, incrementando los riesgos sobre su salud. En estas zonas, se aplican aún pesticidas organoclorados derivados del DDT que afectan la condición física de las aves y producen efectos en su salud (Jiménez et al. 2005). Estos compuestos podrían producir afectaciones a la condición física de los individuos lo que puede producir efectos en su supervivencia. Este factor puede influir en las causas del declive poblacional de especies en Norteamérica por la condición del hábitat en las áreas de migración (Farmer \& Smith 2009, Smallwood et al. 2009).

Sin embargo, más estudios deben enfocarse a los movimientos de las especies utilizando marcaje individual y telemetría. Realizar el seguimiento de individuos de ambas especies por estas técnicas durante el año permitiría determinar la manera en que las especies utilizan los diferentes ambientes. Asimismo, se deben evaluar los efectos que tengan los pesticidas (e.g. compuestos organoclorados) en poblaciones residentes y migratorias. Lo anterior puede tener profundas implicaciones para la conservación de estas especies. 
Agradecimientos. Agradecemos el apoyo en campo de Abelino Cota, Adrian Munguía, Alejandra Partida, Franco Cota y Bruno Granados. Al CONACyT por la beca de doctorado otorgada a RTH (número de registro 98227). El apoyo económico de los proyectos SEMARNAT-CONACyT (2002-C01-0317, 0000023861), SEP-CONACyT (155956) concedidos a RRE permitió realizar esta investigación. Agradecemos a dos revisores anónimos sus sugerencias que permitieron mejorar el escrito.

\section{LITERATURA CITADA}

Andersen, D.E., O.J. Rongstad \& W.R. Mytton. 1985. Line transect analysis of raptor abundance along roads. Wildlife Society Bulletin, 13:533-539.

Andrén, H. 1994. Effects of habitat fragmentation on birds and mammals in landscapes with different proportions of suitable habitat: a review. Oikos, 71:355-366.

Bierregaard, R.O., T.E. Lovejoy., V.Kapos, A.A. Dos Santos \& R.W. Hutchings. 1992. The biological dynamics of tropical rainforest fragments. A prospective comparison of fragments and continuous forest. BioScience, 42:859-866.

Baicich, P.J. \& C.J.O. Harrison. 2005. Nest, eggs, and nestlings of North American birds. Second Edition, Princeton University Press. Princeton, New Jersey.

Bird, D.M., D. Varland \& J.J. Negro. 1996. Raptors in human landscape. Academic Press, London.

Bird, D.M. \& K.L. Bildstein. 2007. Raptor research and management techniques. Hancock House Publisher, Washington.

Boano, G. \& R. Toffoli. 2002. A line transect survey of wintering raptors in the western Po Plain of northern Italy. Journal of Raptor Research, 36:128-135.

Bosakowsky, T. \& D.G.Smith. 1997. Distribution and species richness of a forest raptor community in relation to urbanization. Journal of Raptor Research, 31: 26-33.

Butet, A., N. Michel, Y. Ranter, V. Comor, L. Hubert-May, J. Nabucet \& Y. Delettre. 2010. Responses of Common buzzard (Buteo buteo) and Eurasian Kestrel (Falco tinnunculus) to land use changes in agricultural landscape of western France. Agriculture, Ecosystem and Environment, 138:152-159.

Campion, D. 2004. Using GIS for identifying features for conserving raptors in altered habitats: A case study in a modified Mediterranean landscape. Pp 153-178. In. R. Rodríguez-Estrella \&. L.A.T. Bojórquez [Eds.]. Spatial Analysis in Raptor Ecology and Conservation. CIBNOR-CONABIO. La Paz, Baja California Sur, México.

Cardador, L., M. Carrete \& S. Mañosa. 2011. Can intensive agricultural landscapes favour some raptor species? The Marsh Harrier in north-eastern Spain. Animal Conservation, 14:382-390.

Carrete, M., J.L. Tella, G. Blanco \& M. Bertellotti. 2009. Effects of habitat degradation on the abundance, richness and diversity of raptor across neotropical biomes. Biological Conservation, 142:2002-2011.

Chamberlain, D.E., R.J. Fuller, R.G.H. Bunce, J.C. Duckworth \& M. Shrubb. 2000. Changes in the abundance of farmland birds in relation to the timing of agricultural intensification in England and Wales. Journal Applied Ecology, 37:771-788.

Clark, W.S. \& B.K. Wheeler. 2001. Hawks of North America, second ed. Houghton Mifflin, Boston, MA. U.S.A.

Corral, J.A., G. Medina-García, R. Meza-Sánchez, G. Díaz-Padilla \& V. Serrano-Altamirano. 2006. Estadísticas climatológicas básicas del estado de Baja California Sur, periodo 1961-2003. Centro de Investigación del Noroeste, INIFAP. México.

Crowder, M. \& D. Hand. 1990. Analysis of repeated measures. Chapman and hall. New York. USA.

Davies, K.F. \& C.R. Margules. 1998. Effects of habitat fragmentation on carabid beetles: experimental evidence. Journal of Animal Ecology, 67:460-471. 
Davison, M.A. \& J.W. Fitzpatrick. 2010. Role of human-modified habitat in protecting specialist species: A case study in the threatened Florida Scrub-Jay. Biological Conservation, 143:2815-2822.

Devictor, V., R. Julliard \& F. Jiguet. 2008. Distribution of specialist and generalist species along spatial gradients of habitat disturbance and fragmentation. Oikos, 117:507-514.

Donazar, J.A., O. Ceballos, A. Travaini \& F. Hiraldo. 1993. Roadside raptor surveys in the Argentinean Patagonia. Journal of Raptor Research, 27:106-110.

Fahrig, L. 1997. Relative effects of habitat loss and fragmentation on population extinction. Journal of Wildlife Management, 61:603-610.

Fahrig, L. 2003. Effects of habitat fragmentation on biodiversity. Annual Review of Ecology and Systematics, 34:487-515.

Farmer, C.J. \& J.F. Smith. 2009. Migration monitoring indicates widespread declines of American Kestrel (Falco sparverius) in North America. Journal of Raptor Research, 43:263-273.

Ferguson, H.L. 2004. Relative abundance and diversity of winter raptors in Spokane County, Eastern Washington. Journal of Raptor Research, 38: 181-186.

Filloy, J. \& M.I. Bellocq. 2007. Respuesta de las aves rapaces al uso de la tierra: un enfoque regional. Hornero, 22:131-140.

Fuller, M.R. \& J.A. Mosher. 1987. Raptor Survey Techniques. Pp 37-65. In. B.A. Millsap, K.W Cline \&. D.M. Bird [Eds.]. Raptor Management Techniques Manual. National Wildlife Federation. Washington, D.C.

Giraudo, A.R., S.D. Matteucci, J. Alonso, J. Herrera \& R.R. Abramson. 2008. Comparing bird assemblages in large and small fragments of the Atlantic forest hotspots. Biodiversity and Conservation, 17:1251-1265.

Goldstein, M.I. \& T.J. Hibbitts. 2004. Summer roadside raptor surveys in the Western Pampas of Argentina. Journal of Raptor Research, 38:152-157.

Hobson, K. A. \& E. Bayne. 2000. Effects of forest fragmentation by agriculture on avian communities in the southern boreal mixed woods of Western Canada. The Wilson Bulletin, 112:373-387.

Hoffman, S. W. \& J.P. Smith. 2003. Population trends of migratory raptors in western North America, 1997-2001. Condor, 105:397-419.

INEGI. 1995. Síntesis geográfica del estado de Baja California Sur. Aguascalientes, México.

Jensen, W.J., M.S. Gregory, G.A. Baldassarre, F.J. Vilella \& K.L. Bildstein. 2005. Raptor abundance and distribution in the Llanos wetlands of Venezuela. Journal of Raptor Research, 39:417-428.

Jiménez, B., Rodríguez-Estrella, R., Merino, R., Gómez, G., Rivera-Rodríguez L., González, M. J., Abad, E. \& J. Rivera. 2005. Results and evaluation of the first study of organoclorine contaminants (PCDDs, PCDFs, PCBs, and DDTs), heavy metals and metalloids in birds from Baja California, México. Environmental Pollution, 133: 139-146

Jullien, M. \& J.M. Thiollay. 1996. Effects of rain forest disturbance and fragmentation: comparative change of the raptor community along natural and human-made gradients in French Guiana. Journal of Biogeography, 23: 7-25.

Kattan, G. H., Álvarez-López, H. \& M. Giraldo. 1994. Forest fragmentation and bird extinctions: San Antonio eighty years later. Conservation Biology, 8:138-146.

Kim, D. H., R.D. Slack \& F. Chávez-Ramírez. 2008. Impact of El Niño southern oscillation events on the distribution of wintering raptors. Journal of Wildlife Management, 72:231-239.

Kirk, D.A. \& J.E.P. Curral. 1994. Habitat associations of migrant and resident vultures in central Venezuela. Journal of Avian Biology, 25:327-337.

Kirk, D.A. \& A.G. Gosler. 1994. Body condition varies with migration and competition in migrant and resident south American Vultures. The Auk, 111:933-944. 
Tinajero \& Rodríguez-Estrella: Efectos de fragmentación del matorral en aguililla y cernícalo

Knick, S.T. \& J.T. Rotenberry. 1995. Landscape characteristics of fragmented shrubsteppe habitats and breeding passerine birds. Conservation Biology, 9:1059-1071.

Laurance, W.F., P. Delam, S.G. Laurance, H.L. Vasconcelos \& T.E. Lovejoy. 2000. Conservation: Rainforest fragmentation kills big trees. Nature, 404:836.

Lish, J.W. \& L.J. Burge. 1995. Population characteristics of Red-tailed Hawks wintering of tallgrass prairies in Oklahoma. The Southwestern Naturalist, 40:174-179.

Marin, M. \& J. Schmitt. 1996. A road survey of raptors through western Mexico. Ornitologia Neotropical, 7:173-175.

McCrary, M.D., R.L. McKernan, W.D. Wagner \& R.E. Landry. 1985. Roadside raptor census in the San Jacinto Valley of Southern California. Western Birds, 16:123-130.

Newton, I. 1979. Population ecology of raptors. Ed. Buteo Books. South Dakota U.S.A. 399 pp.

Nikolov, S., S. Pasov \& N. Kambourova. 2006. Density, number and habitat use of Common Buzzard (Buteo buteo) wintering in the lowlands of Bulgaria. Buteo, 15: 39-47.

Pavez, E.F., G.A. Lobos \& F.M. Jaksic. 2010. Cambios de largo plazo en el paisaje y los ensambles de micromamíferos y rapaces en Chile Central. Revista Chilena de Historia Natural, 83: 99-111.

Preston, C. R. 1990. Distribution of raptor foraging in relation to prey biomass and habitat structure. The Condor, 92:107-112.

Preston, C.R. \& R.D. Beane. 1993. Red-tailed Hawk (Buteo jamaicensis). In: The birds of North America (A. Poole and F. Gil, Eds). Academy of Natural Sciences, Philadelphia, Pennsylvania y The American Ornithologist Union, Washington D.C.

Robinson, G.R., R.D. Holt, M. S. Gaines, S. P. Hamburg, M. L. Johnson, H.S. Fitch \& E. A. Martinko. 1992. Diverse and contrasting effects of habitat fragmentation. Science, 257:524-526.

Rodríguez-Estrella, R. 2007. Land use changes affect distributional patterns of desert birds in the Baja California peninsula, Mexico. Diversity and Distributions, 13:877-889.

Rodríguez-Estrella, R., J. A. Donázar y F. Hiraldo. 1998. Raptors as indicators of environmental change in the scrub habitat of Baja California Sur, Mexico. Conservation Biology, 12:921-925.

Ruelas, I.E., L.J. Goodrich \& S.W. Hoffman. 2010. Cambios en las poblaciones de aves rapaces migratorias en Veracruz, México, 1995-2005. Acta Zoológica Mexicana (ns), 26:495-525.

Salinas-Zavala, C.A., S.E. Lluch-Cota \& I. Fogel. 2006. Historia del desarrollo del cultivo invernal de trigo en cinco distritos de riego en el desierto de Sonora, México. Interciencia, 31:254-261.

Santos, T. \& J.L. Telleria. 2006. Pérdida y fragmentación del hábitat: efecto sobre la conservación de las especies. Ecosistemas, 2:3-12.

Santos, T., J. L.Telleria, \& R. Carbonell. 2002. Bird conservation in fragmented Mediterranean forests of Spain: effects of geographical location, habitat and landscape degradation. Biological Conservation,105:113-125.

Sauer, J,R., J.E. Hines \& J. Fallon. 2008. The north American breeding bird survey, result and analysis 1966-2004. Version 2005. USGS Patuxent Wildlife Research Center, Laurel Maryland. (http:/ www.mbr-pwrc.usgs.gov/)

Seavy, N. E. \& C.K. Apodaca. 2002. Raptor abundance and habitat use in a highly-disturbed-forest landscape in western Uganda. Journal of Raptor Research, 36:51-57

Sergio, F., I. Newton \& L. Marchesi. 2005. Top predators and biodiversity. Nature, 436:192.

Sergio, F., T. Caro, D. Brown, B Clucas, J. Hunter., J. Ketchum, K. McHugh \& F. Hiraldo. 2008. Top predators as conservation tools: ecological rationale, assumptions, and efficacy. Annual Review of Ecology, Evolution and Systematics, 39:1-19.

Sibley, D.A. 2000. National Audubon Society, the Sibley Guide to Birds. Alfred A. Knopf, Inc., New York. 
Siriwardena, G. M., S.R. Baillie, S.T. Buckland, R.M. Fewster, J.H. Marchant \& J.D. Wilson. 1998. Trends in the abundance of farmland birds: a quantitative comparison of smoothed common birds census indices. Journal of Applied Ecology, 35: 24-43.

Smallwood, J.A., M.F. Causey, D.H. Mossop, J.R. Klucsarits, B. Robertson, S. Robertson, J. Manson, M.J. Maurer, R.J. Melvin, R.D. Dawson, G.R. Bortolotti, J.W. Parrish, T.F. Breen \& K. Body. 2009. Why are American Kestrel (Falco sparverius) populations declining in North America? Evidence from nest-box programs. Journal of Raptor Research, 43:274-282.

Southwood, T.R.E. \& P.A. Henderson. 2000. Ecological methods. Third Edition. Blackwell Science. Oxford, London.

Sullivan, L.B. \& Wood C.L. 2005. The changing Season: Plea for the Common Birds. North American Birds, 59:18-30.

Tella, J.L. \& Forero, M.G. 2000. Farmland habitat selection of wintering lesser kestrels in a Spanish pseudosteppe: implications for conservation strategies. Biodiversity and Conservation, 9:433-441.

Tejas, A., R. Servín \& S. Gallina. 1991. Delimitación, zonificación y tenencia de la tierra. Pages 5368. In: L. Arriaga [ed.], La Reserva de la Biosfera El Vizcaíno en la península de Baja California. Publicaciones CIB, La Paz, B.C.S., México.

Thiollay, J.M. 1989. Censusing of diurnal raptors in a primary rain forest: comparative methods and species detectability. Journal of Raptor Research, 23:72-84.

Thiollay, J. 1993. Response of a raptor community to shrinking area and degradation of tropical rain forest in the south western Ghats (India). Ecography, 16:97-110.

Thomas, L., S.T. Buckland, E.A. Rexstad, J.L. Laake, S. Strindberg, S.L. Hedley, J.R.B. Bishop, T.A. Marques \& P. Burnham. 2010. Distance software: design and analysis of distance sampling surveys for estimating population size. Journal of Applied Ecology, 47:5-14.

Turner, I. M. 1996. Species loss in fragments of tropical rain forest: a review of the evidence. Journal of Applied Ecology, 33:200-209.

Valencia, D., D. Leal \& E. Barriga. 1980. The influence of agriculture on avian communities near Villavicencio, Colombia. Willson Bulletin, 92:381-389.

Van Horne, B. 1983. Density as a misleading indicator of habitat quality. Journal of Wildlife Management, 74:893-901.

Wiggins, I.L. 1980. Flora of Baja California. Stanford University Press, Stanford CA.USA.

Williams, C.K., R.D. Applegate, R. Scott-Lutz \& D.H. Rush. 2000. A comparison of raptor densities and habitat use in Kansas cropland and rangeland ecosystems. Journal of Raptor Research, 34:203209.

Zar, J.H. 1996. Biostatistical Analysis. Third Ed. Prentice Hall, Princeton N.J.

Zurita, G.A. \& M.I. Bellocq. 2007. Pérdida y fragmentación de la selva Paranaense: efectos sobre las aves rapaces diurnas. Hornero, 22:141-147. 
Tinajero \& Rodríguez-Estrella: Efectos de fragmentación del matorral en aguililla y cernícalo

\section{APÉNDICE 1}

Valores promedio de abundancias relativas (aves/100 km \pm e.s.) de Buteo jamaicensis y Falco sparverius para cada estación y año en el área fragmentada y natural. En negritas probabilidades significativas.

\begin{tabular}{|c|c|c|c|c|c|c|}
\hline & \multicolumn{2}{|c|}{ Área Natural } & \multirow[b]{2}{*}{$F(p)$} & \multicolumn{2}{|c|}{ Área Fragmentada } & \multirow[b]{2}{*}{$\mathrm{F}(\mathrm{p})$} \\
\hline & 2008 & 2009 & & 2008 & 2009 & \\
\hline \multicolumn{7}{|l|}{ Primavera-Verano } \\
\hline Buteo jamaicensis & $1.85 \pm 0.7$ & $1.54 \pm 0.9$ & $0.15(0.7)$ & $1.35 \pm 0.5$ & $1.23 \pm 0.7$ & $0.02(0.86)$ \\
\hline Falco sparverius & $10.18 \pm 1.3$ & $18.52 \pm 3.3$ & $5.4(0.03)$ & $13.97 \pm 2.5$ & $19.75 \pm 3.8$ & $1.06(0.31)$ \\
\hline \multicolumn{7}{|l|}{ Otoño } \\
\hline Buteo jamaicensis & $12.65 \pm 1.5$ & $16.67 \pm 2.2$ & $2.1(0.15)$ & $30.55 \pm 2.7$ & $40.43 \pm 6.8$ & $1.8(0.19)$ \\
\hline Falco sparverius & $32.41 \pm 4.8$ & $52.47 \pm 3.7$ & $11(0.003)$ & $34.5 \pm 2.4$ & $52.47 \pm 2.1$ & $31(<0.0001)$ \\
\hline \multicolumn{7}{|l|}{ Invierno } \\
\hline Buteo jamaicensis & $14.20 \pm 3.0$ & $9.87 \pm 2.8$ & $1.1(0.34)$ & $43.8 \pm 6.9$ & $20.99 \pm 3.8$ & $5.43(0.029)$ \\
\hline Falco sparverius & $25.0 \pm 6.1$ & $25.6 \pm 5.6$ & $0.006(0.9)$ & $23.15 \pm 3.6$ & $29.32 \pm 5.1$ & $1.7(0.19)$ \\
\hline
\end{tabular}

\section{APÉNDICE 2}

Valores promedio de abundancias relativas (aves/100 km \pm e.s.) de Buteo jamaicensis y Falco sparverius para 2008 y 2009. En negritas probabilidades significativas.

\begin{tabular}{llllllll}
\hline & \multicolumn{2}{l}{2008} & & & 2009 & \\
\cline { 2 - 3 } & A.N. & A.F. & $\mathrm{F}(\mathrm{p})$ & & A.N. & A.F. & $\mathrm{F}(\mathrm{p})$ \\
\hline Primavera-Verano & & & & & & \\
Buteo jamaicensis & $1.85 \pm 0.7$ & $1.35 \pm 0.5$ & $0.47(0.5)$ & $1.54 \pm 0.9$ & $1.23 \pm 0.7$ & $0.06(0.80)$ \\
Falco sparverius & $10.18 \pm 1.3$ & $13.97 \pm 2.5$ & $1.72(0.2)$ & $18.52 \pm 3.3$ & $19.75 \pm 3.8$ & $1.13(0.48)$ \\
Otoño & & & & & & \\
Buteo jamaicensis & $12.65 \pm 1.5$ & $30.5 \pm 2.0$ & $33(<0.0001)$ & $16.66 \pm 2.2$ & $40.43 \pm 6.8$ & $10.9(0.003)$ \\
Falco sparverius & $32.41 \pm 4.8$ & $34.57 \pm 2.4$ & $0.16(0.7)$ & $52.47 \pm 3.7$ & $52.47 \pm 2.1$ & $0(1)$ \\
Invierno & & & & & & \\
Buteo jamaicensis & $14.2 \pm 3.1$ & $43.82 \pm 6.9$ & $11(0.003)$ & $9.87 \pm 2.8$ & $20.99 \pm 3.8$ & $5.57(0.028)$ \\
Falco sparverius & $25.0 \pm 6.1$ & $23.15 \pm 3.6$ & $0.31(0.6)$ & $25.62 \pm 5.6$ & $29.32 \pm 5.1$ & $0.24(0.6)$ \\
\hline
\end{tabular}

A.N.: Área natural, A.F.: Área Fragmentada 